\title{
Bioequivalence and Pharmacokinetic Comparison of Two Metformin Immediate-release Formulations in Healthy Volunteers under Fed Conditions
}

\author{
Wen Yao Mak ${ }^{1 *}$, Siew Siew Tan², Jia Woei Wong ${ }^{2}$, Siaw Kuen Chin², Ai Boey Lim², Irene Looi ${ }^{1}$ and Kah Hay Yuen ${ }^{2}$ \\ ${ }^{1}$ Clinical Research Centre, Hospital Seberang Jaya, Jalan Tun Hussein Onn, 13700 Seberang Jaya, Pulau Pinang, Malaysia \\ ${ }^{2}$ Hovid Research Pte Ltd, USM Laboratory Room 024, Kompleks EUREKA, Universiti Sains Malaysia, 11800 USM, Pulau Pinang, Malaysia
}

\begin{abstract}
Background: Type-2 diabetes mellitus is a common metabolic disorder in Malaysia, where metformin is used as the first-line treatment. Since metformin absorption is altered when administered with food, the US FDA guidance on metformin hydrochloride recommended to conduct bioequivalence study in both fasting and fed conditions. In this study, we intended to establish bioequivalence between a locally manufactured metformin formulation with a reference formulation under fed condition.
\end{abstract}

Methods: The study was a single-dose, open label, randomised, two treatments, two periods, cross-over study in 24 healthy volunteers. The washout period was 7 days to allow adequate time for drug elimination from the body. Analysis of plasma metformin concentration was conducted with a validated reverse-phase HPLC with ultraviolet detection procedure. Non-compartmental modal was used to analyse the pharmacokinetic parameters. Tolerability of both formulations was assessed throughout the study.

Results: A total of 24 volunteers were recruited, only 21 had completed the study. Two volunteers dropped out of the study due to personal reason. The third volunteer experienced emesis within 4 hour post dose administration hence was not included into the analysis. There was no significant difference observed between the two formulations for the parameters assessed. The $95 \%$ confidence interval for the ratio of test over reference parameters were within the acceptance limits of $80.00 \%$ to $125.00 \%$ : $C_{\max } 0.8864-1.0554, A \cup C_{0-t} 0.8835-1.0184, A C_{0-\infty} 0.8933-1.0229$. No serious adverse event was observed.

Conclusions: The test metformin $250 \mathrm{mg}$ formulation was bioequivalent to the reference formulation.

Keywords: Bioequivalence; Pharmacokinetics; Metformin; Type II diabetes mellitus

\section{Introduction}

Metformin is a biguanide which is commonly used to treat type 2 diabetes mellitus. It is recommended by the National Institute for Health and Care Excellence (NICE) and the Malaysian Ministry of Health as the first-line treatment for type 2 diabetic patients after lifestyle modification fails to control the blood glucose level $[1,2]$.

Metformin displays altered absorption when co-administered with food. Limited literature was found on metformin pharmacokinetics under fed condition, but it is known that food will decrease the rate and extent of metformin absorption. The Summary of Product Characteristic of Glucophage states the co-administration of Glucophage with food will reduce the maximum plasma concentration $\left(\mathrm{C}_{\max }\right)$ by approximately $40 \%$; total area under the plasma concentration versus time curve (AUC) reduced by $25 \%$, and 35 -minutes delay in time to reach $\mathrm{C}_{\max }\left(\mathrm{T}_{\max }\right)$ [3]. In general, metformin has poor absolute bioavailability of approximately $50 \%$ to $60 \%$. The lack of dose proportionality with increasing dose suggests that the absorption of metformin is mediated by an active, saturable absorption process [3-5]. Metformin does not undergo hepatic metabolism, and it is excreted unchanged in the urine, with a plasma elimination half-life between 4.0 and 8.7 hours $[3,4,6]$.

Common side effects reported with metformin include diarrhoea, flatulence, abdominal pain, nausea and vomiting, and headache [1]. Lactic acidosis is rare, happening only at a rate of approximately 0.03 cases per 1,000 patient-years $[1,3]$.
According to the United States Food and Drug Administration's (FDA's) Guidance on Metformin Hydrochloride, it is recommended to conduct the bioequivalence study in both fasting and fed condition [7]. However the document allows flexibility to the design of the study if appropriately justified. We had completed the metformin bioequivalence study under fasting condition [8] and the current study was conducted under fed condition.

The objective of the current study is to investigate the rate and extent of absorption of a generic metformin $250 \mathrm{mg}$ immediate-release (IR) formulation, manufactured by Hovid Ltd (Malaysia), against that of a reference formulation (manufactured by Sunward Pharmaceutical Pte Ltd, Singapore) to establish bioequivalence in healthy volunteers under fed condition.

\section{Methods}

The study was conducted in accordance to the World Medical

*Corresponding author: Wen Yao Mak, Clinical Research Centre, Hospita Seberang Jaya, Jalan Tun Hussein Onn, 13700 Perai, Pulau Pinang, Malaysia Tel: 04-3827333 Ext 511; Fax: 04-3902192; E-mail: makwy@crc.gov.my

Received April 10, 2015; Accepted May 14, 2015; Published May 21, 2015

Citation: Mak WY, Tan SS, Wong JW, Chin SK, Lim AB, et al. (2015) Bioequivalence and Pharmacokinetic Comparison of Two Metformin Immediaterelease Formulations in Healthy Volunteers under Fed Conditions. J Bioequiv Availab 7: 184-188. doi:10.4172/jbb.1000236

Copyright: $\odot 2015$ Mak WY, et al. This is an open-access article distributed unde the terms of the Creative Commons Attribution License, which permits unrestricted use, distribution, and reproduction in any medium, provided the original author and source are credited. 
Association (WMA) Declaration of Helsinki and Malaysian Good Clinical Practice Guideline. The study protocol was reviewed and approved by the Malaysian Medical Research Ethic Committee. All investigators involved in the study were certified under Malaysia Good Clinical Practice guideline.

The study was conducted at the Clinical Trial Unit, Seberang Jaya Hospital, Penang, Malaysia while the Bioanalysis was conducted at Hovid Research Pte Ltd, Universiti Sains Malaysia, Penang, Malaysia.

\section{Study population}

Male volunteers who were between the age of 21 to 55 years old, with body mass index between 18.5 to $29.9 \mathrm{~kg} / \mathrm{m}^{2}$ or within $20 \%$ ideal body weight for height and build according to the Metropolitan Life Insurance Company Standards, and were in good health and physical condition as determined by medical history and laboratory tests were eligible to participate in the study. The laboratory tests included renal function tests, liver function tests, full blood count, fasting blood glucose test and 12-lead electrocardiogram.

Volunteers who met the exclusion criteria were not recruited into the study. These included any volunteers who did not fulfill the inclusion criteria, those who had significant clinical deviation from normal, a history or suspicion of drug or alcohol abuse, hypersensitivity to metformin, participated in other bioequivalence studies or donated blood in the past 8 weeks, unable to understand or comply to elements of the protocol, unable to give consent, or who smoked more than ten cigarettes a day.

Informed consent was obtained from all volunteers before any procedures pertaining to the study were performed. All volunteers were screened and recruited at the clinical site by certified clinicians.

\section{Sample size calculation}

The study sample size was calculated with the intrasubject coefficient of variation (CV) [9]. Previous studies of metformin immediate release tablets as reported by Yuen et al (2000) indicated that the values for intrasubject CV were less than $25 \%$ for $\mathrm{C}_{\max }, \mathrm{AUC}_{0-\mathrm{t}}$ and $\mathrm{AUC}_{0-\infty}[10]$. Therefore a total of 24 subjects were required to achieve a statistical power of $80 \%$ (assuming that the $\mu_{\mathrm{T}} / \mu_{\mathrm{R}}$ did not deviate by more than $5 \%)$

\section{Study design}

The study was an open label, randomized, single dose, two treatments, two periods, cross-over study in 24 healthy volunteers. The washout period between two study periods was at least 7 days to allow adequate elimination of drug from the body. Prior to each dosing day, the volunteers were quarantined in the clinical site, and were required to undergo a 10 -hour fasting period. During these periods, the volunteers were allowed access to plain water only.

All subjects were given a standardized, high fat, high calorie breakfast 30 minutes prior to drug administration. The subjects were randomized into one of two groups, where they received the test or reference formulation on either first or second study period. The drug was administered with $240 \mathrm{ml}$ of plain water. No intake of water was allowed one hour prior and after drug administration except for the amount that was used for drug administration. Subsequently, all subjects received $60 \mathrm{ml}$ of $20 \%$ glucose solution on hourly basis until 3 hours post dosing. Standardized meals were provided to subjects at $4^{\text {th }}$ and $10^{\text {th }}$ hour post dose for both study periods. Subjects were monitored and prohibited from consuming additional food or drinks, any alcoholic or caffeine containing beverages, and tobacco use.
To maintain consistency, all test and reference drugs were taken from the same manufacturing batch with identical expiry date.

\section{Blood sampling and analysis}

Blood samples of $5 \mathrm{ml}$ volume were collected at 0 (pre-dose), $0.5,1$, $1.5,2,2.5,3,4,5,6,8,10,12,16$, and 24 hour post dose administration, via an in-dwelling cannula placed at the forearms or via venipuncture. A total of approximately $75 \mathrm{ml}$ blood were taken from each subject during each study period. The blood samples were collected into vacutainers (containing sodium heparin as anticoagulant), and were immediately centrifuged for 15 minutes at $3500 \mathrm{rpm}$. The resulting plasma samples were transferred to glass containers and kept frozen at $-40^{\circ} \mathrm{C}$ until analysis.

Metformin plasma concentration levels were analyzed with reverse-phase high performance liquid chromatography (HPLC) with ultraviolet detection. The HPLC system comprised of Waters 600E Multisolvent Delivery System (Maple Street Milford, USA), Waters 2487 Dual Channel UV-VIS Detector (Maple Street Milford, USA), Waters 717Plus Autosampler (Maple Street Milford, USA), and the data acquisition and analysis software, Waters Empower ${ }^{\mathrm{TM}} 2$ Data Software (Maple Street Milford, USA).

The chromatographic separation was performed with a ZORBAX RX-SIL (250 x $4.6 \mathrm{~mm}$ id, $5 \mu$ ) (Agilent Technologies, USA) analytical column, which is fitted with a refillable guard column $(2 \mathrm{~mm} \times 2 \mathrm{~cm})$ (Upchurch Scientific, Oak Harbour, USA) packed with Perisorb RP$18(30-40 \mu \mathrm{m}$, pellicular). The mobile phase consisted a mixture of $10.0 \%$ acetonitrile in $0.03 \mathrm{M}$ diammonium hydrogen phosphate buffer, adjusted to $\mathrm{pH} 6.0$ with $85 \%$ phosphoric acid. The flow rate was set at $1.0 \mathrm{ml} / \mathrm{min}$ isocratically. Detector was operated at $234 \mathrm{~nm}$ and the sensitivity was set at 2.0000 AUFS. The injection volume was $50 \mu \mathrm{l}$ and the samples were quantified using peak area.

\section{Safety assessment}

Clinicians were present at the clinical site for up to 12 hours post dose, and were responsible to monitor the vital signs of all subjects. Readings of blood pressure, pulse rate and temperate were taken for up to 5 times throughout each study period. Before subjects were released from the clinical site, a last vital signs reading was taken to ensure the safety of subjects.

\section{Pharmacokinetic and statistical method}

The pharmacokinetic analysis only included data collected from subjects who completed both study period. Data from subjects who experienced emesis or vomiting within 4 hours of dose administration was excluded as well. For blood sampling time, a 5\% deviation from time planned was allowed before it was recorded as a protocol deviation. If the sampling time exceeded the $5 \%$ limit, the actual sampling time was used to calculate the pharmacokinetic parameters.

Four pharmacokinetic parameters were derived from the plasma concentration versus time profile: the maximum plasma concentration $\left(\mathrm{C}_{\max }\right)$, the time to reach maximum plasma concentration $\left(\mathrm{T}_{\max }\right)$, the area under the plasma concentration-time curve from time zero to the last measurable concentration $\left(\mathrm{AUC}_{0-\mathrm{t}}\right)$, and the total area under the plasma concentration-time curve $\left(\mathrm{AUC}_{0-\infty}\right)$. The values of $\mathrm{C}_{\max }$ and $\mathrm{T}_{\max }$ were obtained directly from the plasma concentration-time curve, while the values of $\mathrm{AUC}_{0-\mathrm{t}}$ were calculated using the trapezoidal formula. $\mathrm{AUC}_{0-\infty}$ was calculated by adding the value of $\mathrm{AUC}_{0-\mathrm{t}}$ with the value of $A U C_{t-\infty}$, the area under the concentration-time curve from time $\mathrm{t}$ to infinity. Values of $\mathrm{AUC}_{\mathrm{t}-\infty}$ were derived by dividing 
the last measurable plasma concentration $\left(\mathrm{C}_{\mathrm{t}}\right)$ with the elimination rate constant $\left(\mathrm{k}_{\mathrm{e}}\right)$ [11]. According to the Malaysian Guideline for the Conduct of Bioavailability and Bioequivalence Studies, the $\mathrm{AUC}_{\mathrm{t}-\infty}$ should not be more than $20 \%$ of $\mathrm{AUC}_{0-\infty}$ [12].

Metformin elimination rate constant, $\mathrm{k}_{\mathrm{e}}$, was estimated from the terminal slope of the individual plasma concentration-time curve which underwent natural logarithmic (ln) transformation and the application of linear regression [13]. At least three values were used in the estimation. The value of $k_{e}$ was then use to calculate metformin half-life $\left(\mathrm{t}_{1 / 2}\right)$ with the formula $\mathrm{t}_{1 / 2}=\ln 2 / \mathrm{k}_{\mathrm{e}}$.

Statistical analysis was performed with commercial software, EquivTestPK from Statistical Solution (Cork, Ireland). The analysis of variance (ANOVA) was used to analyze the values of $\mathrm{C}_{\max }, \mathrm{AUC}_{0-\mathrm{t}}$, $\mathrm{AUC}_{0-\infty}$ and $\mathrm{k}_{\mathrm{e}}$ obtained from the two study periods. ANOVA was used because it can distinguish the effects due to subjects, periods, treatments and sequences difference [14]. Wilcoxon Signed Rank Test for paired samples was used to analyze $\mathrm{T}_{\max }$ values. The statistical analysis was consistent with the recommendation by the Malaysian Guideline for the conduct of Bioavailability and Bioequivalence Studies.

Bioequivalence between the two formulations was determined based on the $90 \%$ confidence interval of the ratio of $\mathrm{C}_{\max }, \mathrm{AUC}_{0-\mathrm{t}}$ and $\mathrm{AUC}_{0-\infty}$ of the generic formulation (Diabetmin $250 \mathrm{mg}$ tablet) over that of the reference formulation (Sunward Metformin $250 \mathrm{mg}$ ). There $\alpha$-error was maintained at $5 \%$ level of significance [15].

The acceptable range to conclude bioequivalence, as reported by US FDA and the Malaysian Ministry of Health, is $80.00-125.00 \%$ for $\mathrm{AUC}_{0-\mathrm{t}}, \mathrm{AUC}_{0-\infty}$ and $\mathrm{C}_{\max }[12,16]$. In addition, the Malaysian guideline allowed a wider range for $\mathrm{C}_{\max }$ if it was appropriately justified, taking into consideration of safety and efficacy issues [12].

\section{Analysis of plasma metformin concentration}

The frozen plasma samples were thawed and $250 \mu \mathrm{l}$ aliquot was transferred to a glass tube. This was followed by the addition of 250 $\mu \mathrm{l}$ of $2.5 \mu \mathrm{g} / \mathrm{ml}$ cimetidine solution, $250 \mu \mathrm{l}$ of $7.5 \mathrm{M}$ sodium hydroxide solution and $5 \mathrm{ml}$ of extraction solvent (butanol:hexane; 1:1; v/v). Cimetidine was used as the internal standard in this instance. The mixture was vortex-mixed for 60 seconds, and centrifuged for 10 minutes at $3500 \mathrm{rpm}$. The organic layer was subsequently transferred to another glass tube, and $250 \mu \mathrm{l}$ of $1 \mathrm{M}$ acetic acid was added. This mixture was vortex-mixed again for 60 seconds and centrifuged for 10 minutes at $3500 \mathrm{rpm} .50 \mu \mathrm{l}$ of the lower aqueous layer was then injected into the column.

Cimetidine had similar retention time to metformin, which were 9.0 and 12.3 minutes respectively. The accuracy and precision of the analytical method was assessed prior to sample analysis. Accuracy was expressed as the percentage of measured concentrations over that of the spiked value; precision was denoted coefficient of variation. For within-day validation, accuracy did not deviate by more than $\pm 13.0 \%$, and at the limit of quantification accuracy did not deviate by more than $\pm 17 \%$. For between-day validation, accuracy did not deviate by more than $\pm 7 \%$ for all concentrations measured. The coefficient of variation (precision) was shown to be less than $4.0 \%$ for all concentrations measured during within-day validation; it was less than $9.0 \%$ for all concentrated studied during between-day validation as well. The absolute recovery of the metformin was above $87 \%$. The method was deemed selective from the lack of significant endogenous peak from six different sources of blank plasma at the retention time of metformin and the internal standard.
The stability of metformin was assessed, where the freeze-thaw stability tests showed metformin was stable in plasma for at least 3 freeze-thaw cycles. Metformin was stable in plasma which was stored at $-15^{\circ} \mathrm{C}$ to $-22^{\circ} \mathrm{C}$ for two months, a period long enough to complete all sample analysis. Short term stability studies indicated that metformin in plasma was stable for 6 hours under room temperature; and metformin in reconstituted solution was stable for 10 hours under room temperature. Drug solutions of metformin and cimetidine were stable for 2 months under refrigerated condition and stable for 9 hours under room temperature.

\section{Results}

A total of 24 subjects were recruited, but only 21 had completed the study. The demographic distribution of the subject population was shown in Table 1. One subject (no. 24) failed to turn up for the first study period due to personal reason; a second subject (no. 1) did not attend the second study period, and was excluded from the study; subject no. 16 completed both study periods but experienced emesis within 4 hours post-dose, hence the data collected was not included in the analysis. All recruited subjects were male, with a median age of 26 years (interquartile range of 9 years), and median BMI $24.3 \mathrm{~kg} / \mathrm{m}^{2}$ (interquartile range of $2.9 \mathrm{~kg} / \mathrm{m}^{2}$ ) (Table 1 ).

There was no significant deviation from the protocol reported. Only minor deviations were observed, and listed as below:

(a) The blood sampling times for subject no.8 (S8) at 0.5 and 1.0 hour were delayed during the first study period. The actual times of blood sampling were used in the pharmacokinetic calculation.

(b) New silica column (no.36) was used for chromatographic separation, and the ratio of mobile phase was changed to allow better peak separation. The new composition of the new mobile phase was acetonitrile:0.03 M diammonium hydrogen phosphate (10:90; v/v), and the new retention for metformin and cimetidine were 9.0 and 12.3 minutes respectively. Partial validation was conducted.

During the bioanalysis of plasma samples, a new silica column was used for the chromatographic separation. The new column contained similar packing and of similar dimension and brand (Zorbax RX-SIL, $250 \times 4.6 \mathrm{~mm}$ id, $5 \mu$ ) (Agilent Technologies, USA). This resulted in significant shifts in the retention times of metformin and cimetidine (internal standard), and satisfactory separation of the two analyte peaks could not be attained. Modification to the mobile phase composition was conducted to ensure the analytes peaks were well-resolved. No failed analytical run and re-assay occurred during the analysis.

Partial validation of the analytical method was allowed where minor changes were made to methods which were already validated $[17,18]$. A partial validation consisting of a within-day run precision and accuracy was conducted to ensure the change in mobile phase did not affect the performance of the method. The mean measured concentration values did not deviate by more than $\pm 13.0 \%$ from the actual concentrations

\begin{tabular}{|l|c|}
\hline Characteristic & Value \\
\hline Age, $\mathrm{y}$ & 26 \\
Median & 9 \\
Interquartile range & \\
\hline BMI, $\mathrm{kg} / \mathrm{m}^{2}$ & 24.3 \\
Median & 2.9 \\
\hline Interquartile range & \\
\hline Ethnicity, \% & $20(95.2)$ \\
Malay & $1(4.8)$ \\
Indian & \\
\hline
\end{tabular}

Table 1: Demographic characteristic of study volunteers $(n=21)$. 
Citation: Mak WY, Tan SS, Wong JW, Chin SK, Lim AB, et al. (2015) Bioequivalence and Pharmacokinetic Comparison of Two Metformin Immediaterelease Formulations in Healthy Volunteers under Fed Conditions. J Bioequiv Availab 7: 184-188. doi:10.4172/jbb.1000236

except for concentration at the limit of quantification, where the measured value did not deviate by more than $\pm 17.0 \%$ from the actual concentration. The values of coefficient of variation were less than $4.0 \%$ for all concentrations.

No severe adverse reaction was reported or observed throughout the study periods. One subject (no.16) experienced emesis and mild nausea after ingestion of the reference formulation, which could be drug related; another subject experienced fever after administration of the reference product. However this was deemed not drug related.

The mean values of plasma concentration over time for the test and reference formulations were shown to be comparable to each other, as seen in Figure 1.

To conclude bioequivalence, the $90 \%$ confidence interval of the ratio, for the three above mentioned parameters, of the test formulation over the reference formulation should be within $80.00 \%$ to $125.00 \%$. In the present study, it was found that the ratio of $\mathrm{AUC}_{0-\mathrm{t}}, \mathrm{AUC}_{0-\infty}$ and $\mathrm{C}_{\max }$ were between $0.8835-1.0184,0.8933-1.0229$ and $0.8864-1.0554$. All ratios were within the acceptable limits to conclude bioequivalence (Table 2).

The value of $\mathrm{T}_{\max }$ showed no statistical significance between the test and reference formulation, as demonstrated by the Wilcoxon Signed Rank Test $(\mathrm{p}=0.0979)$. There were no significant difference found in the values of $\mathrm{k}_{\mathrm{e}}(\mathrm{p}=0.3776)$ and $\mathrm{t}_{1 / 2}(\mathrm{p}=0.1731)$ as well.

The intra-subject variation was estimated, using the mean square error of the ANOVA analysis for $\mathrm{AUC}_{0-\mathrm{t}}, \mathrm{AUC}_{0-\infty}$ and $\mathrm{C}_{\max }$, had coefficient of variation values of $18.8 \%, 17.0 \%$ and $22.3 \%$ respectively. Based on these values, the study sample size of 21 subjects was deemed sufficient to provide a power of approximately $80 \%$ to conclude bioequivalence between two formulations, when $\alpha=0.05$ [19]. The inter-subject variation had coefficient of variation of $28.8 \%, 27.4 \%$ and $28.7 \%$ for three pharmacokinetic parameters.

\section{Discussion}

The fed bioequivalence study was required by the Malaysian National Pharmaceutical Control Bureau as part of the dossier for Diabetmin (Metformin) $250 \mathrm{mg}$ immediate-release tablet application for marketing authorisation. Similar recommendation was made by US FDA's Guidance on Metformin Hydrochloride, which suggested to run bioequivalence study under both fed and fasting condition. However the guidance did allow alternative approach to the conduct of the study [7].

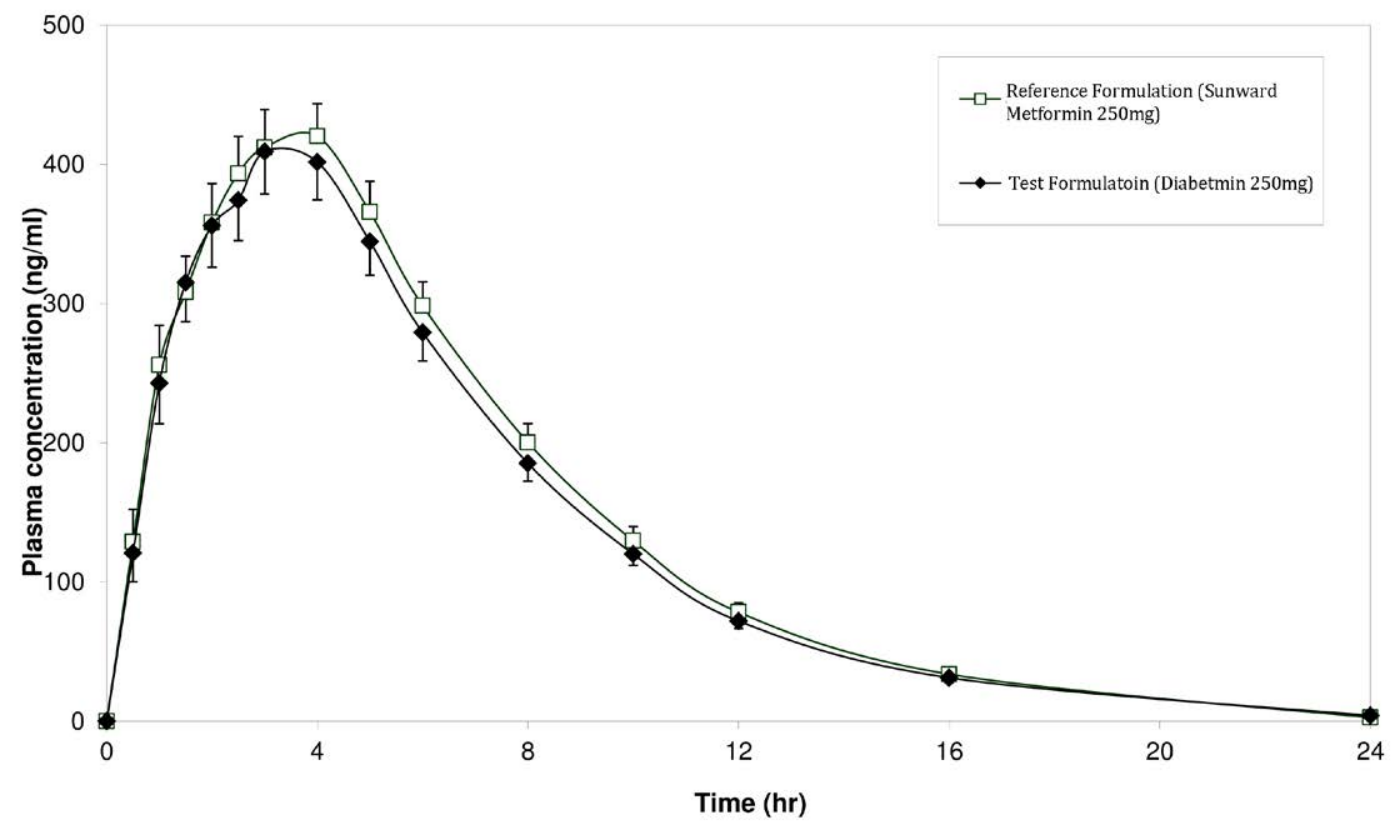

Figure 1: Mean plasma concentration of Metformin $250 \mathrm{mg}$ after dose administration of either the test Diabetmin tablet or the reference Sunward Metformin tablet, under fed condition, in 21 healthy voulnteers.

\begin{tabular}{|c|c|c|}
\hline Pharmacokinetic Parametre & $\begin{array}{l}\text { Diabetmin 250mg tablet (test) } \\
\text { Mean } \pm \text { sd }\end{array}$ & $\begin{array}{c}\text { Sunward Metformin 250mg (reference) } \\
\text { Mean } \pm \text { sd }\end{array}$ \\
\hline $\mathrm{C}_{\max }$ & $434 \pm 152.8$ & $447 \pm 119.7$ \\
\hline$A \cup C_{0-t}$ & $3097 \pm 925.4$ & $3252 \pm 802.6$ \\
\hline $\mathrm{AUC}_{0-\infty}$ & $3284 \pm 935.4$ & $3428 \pm 826.9$ \\
\hline $\mathrm{T}_{\max }(\mathrm{hr})$ & $3.5 \pm 0.71$ & $2.9 \pm 1.05$ \\
\hline $\mathrm{T}_{1 / 2}(\mathrm{hr})$ & $3.8 \pm 1.29$ & $3.5 \pm 0.74$ \\
\hline $\mathrm{K}_{\mathrm{e}}\left(\mathrm{hr}^{-1}\right)$ & $0.2 \pm 0.06$ & $0.2 \pm 0.05$ \\
\hline
\end{tabular}

sd, standard deviation; $\mathrm{AUC}_{0-\mathrm{t}}$, area under the plasma-concentration curve from dosing to last quantifiable time point; $A \cup \mathrm{C}_{0-\infty}$, area under the plasma-concentration curve from dosing to infinity; $\mathrm{C}_{\max }$, peak plasma concentration

Table 2: Pharmacokinetic parametre of the test Metformin formulation (Diabetmin) versus the reference formulation (Sunward Metformin) under fed condition. mean \pm sd $(n=21)$. 
Citation: Mak WY, Tan SS, Wong JW, Chin SK, Lim AB, et al. (2015) Bioequivalence and Pharmacokinetic Comparison of Two Metformin Immediaterelease Formulations in Healthy Volunteers under Fed Conditions. J Bioequiv Availab 7: 184-188. doi:10.4172/jbb.1000236

We had conducted a similar bioequivalence study under fasting condition using the same batch of test and reference tablets. It was found out that co-administration of food and metformin decreases the value of $\mathrm{C}_{\max }, \mathrm{AUC}_{0-\infty}$, and $\mathrm{T}_{\max }: \mathrm{C}_{\max }$ was reduced by approximately $40 \%$, AUC ${ }_{0-\infty}$ decreased by approximately $32 \%$, and $\mathrm{T}_{\max }$ was delayed by nearly 28 minutes [8]. These values were consistent with findings reported in the literature [4-6].

\section{Conclusion}

The test formulation, Diabetmin $250 \mathrm{mg}$ immediate-release tablet (manufactured by Hovid Ltd, Malaysia) was bioequivalent to the reference formulation Sunward Metformin $250 \mathrm{mg}$ immediate-release tablet (manufactured by Sunward Pharmaceutical Pte Ltd, Singapore).

\section{Acknowledgement}

The authors thank all medical staffs at Clinical Research Centre, Hospital Seberang Jaya who contributed to the study.

\section{Declaration of Personal Interest}

Kah Hay Yuen was the advisor to the R\&D department of Hovid Ltd, the manufacturer of the test formulation. Siew Siew Tan, Jia Woei Wong, Siaw Kuen Chin and Ai Boey Lim were employees to Hovid-Research Sdn Bdn, an independent research company which was affiliated with Hovid Ltd. Wen Yao Mak and Irene Looi did not have any conflict of interest to disclose.

\section{Declaration of Funding Interest}

This study was supported by Hovid Ltd.

\section{References}

1. National Collaborating Centre for Chronic Conditions and the Centre for Clinical Practice (UK) (2014) NICE clinical guideline 87: The management of type 2 diabetes [Internet]. National Institute for Health and Care Excellence (UK).

2. CPG Secretariat Health Technology Assessment Section (Malaysia) (2009). Clinical practice guideline: management of type 2 diabetes mellitus. [Internet]. Putrajaya: Ministry of Health (Malaysia).

3. Glucophage [package insert]. Princeton, NJ: Bristol-Myers Squibb Company; 2009.

4. Scheen AJ (1996) Clinical pharmacokinetics of metformin. Clin Pharmacokinet 30: $359-371$

5. Gong L, Goswami S, Giacomini KM, Altman RB, Klein TE (2012) Metformin pathways: pharmacokinetics and pharmacodynamics. Pharmacogenet Genomics 22: 820-827.
6. Graham GG, Punt J, Arora M, Day RO, Doogue MP, et al. (2011) Clinica pharmacokinetics of metformin. Clin Pharmacokinet 50: 81-98.

7. U.S. Food and Drug Administration (2008) Guidance on metformin hydrochloride. Silver Spring (MD): U.S. Food and Drug Administration 2.

8. Mak WY, Tan SS, Wong JW, Chin SK, Looi I, et al. (2015) Bioequivalence study of two IR metformin formulations: an open-label, randomised, two treatments, two-way crossover study in healthy volunteers. J Bioequiv Bioavailab 7: 82-85

9. Steinijans VW, Sauter R, Hauschke D, Diletti E, Schall R, et al. (1995) Reference tables for the intrasubject coefficient of variation in bioequivalence studies. Int J Clin Pharmacol Ther 33: 427-430.

10. Yuen KH, Wong JW, Yap SP, Billa N (2001) Estimated coefficient of variation values for sample size planning in bioequivalence studies. Int $\mathrm{J}$ Clin Pharmacol Ther 39: 37-40.

11. Weiner D (1981) Design and analysis of bioavailability studies. In Buncher C, Tsay J, editors. Statistics in Pharmaceutical Industry. New York: Marcel Dekker. 205-229.

12. Working Committee for Bioequivalence Studies (2000). Malaysian guidelines for the conduct of bioavailability and bioequivalence studies [Internet]. Kuala Lumpur: Ministry of Health.

13. Gibaldi M, Perrier D (1982) Pharmacokinetics, $2^{\text {nd }}$ (Edn). New York: Marcel Dekker. 145

14. Wagner J (1975) Fundamentals of Clinical Pharmacokinetics. Drug Intelligence, $1^{\text {st }}($ Edn). Hamilton. 285.

15. Schuirmann DJ (1987) A comparison of the two one-sided tests procedure and the power approach for assessing the equivalence of average bioavailability. $\mathrm{J}$ Pharmacokinet Biopharm 15: 657-680.

16. U.S Department of Health and Human Services, Food and Drug Administration, Center for Drug Evaluation and Research (CDER) (2002) Guidance for Industry: Food-effect bioavailability and fed bioequivalence studies [Internet] Silver Spring (MD): U.S. Food and Drug Administration;

17. Committee for Medicinal Products for Human Use (CHMP) (2011) Guideline on bioanalytical method validation [Internet]. London: European Medicines Agency.

18. U.S. Department of Health and Human Services, Food and Drug Administration, Center for Drug Evaluation and Research (CDER) (2001) Guidance fo Industry: Bioanalytical Method Validation [Internet]. Silver Spring (MD): U.S. Food and Drug Administration

19. Diletti E, Hauschke D, Steinijans VW (1992) Sample size determination for bioequivalence assessment by means of confidence intervals. Int $\mathrm{J}$ Clin Pharmacol Ther Toxicol 30 Suppl 1: S51-58. 